(REVIEW ARTICLE)

\title{
Tamoxifen resistance mechanisms in breast cancer treatment
}

\author{
Asima Tayyeb * \\ School of Biological Sciences, University of the Punjab, Lahore 54500, Pakistan.
}

Publication history: Received on 15 October 2020; revised on 24 November 2020; accepted on 25 November 2020

Article DOI: https://doi.org/10.30574/wjarr.2020.8.2.0429

\begin{abstract}
Therapies targeting estrogen receptor (ER) are being widely used to treat ER+ breast cancer patients. Despite early detection and improved survival outcomes, tamoxifen resistance, either intrinsic or acquired-is a major obstacle in effective disease management. Current review will summarize different molecular mechanisms of tamoxifen resistance both intrinsic and acquired in breast cancer treatment. This review not only provides basis to understand the nature of tamoxifen drug resistance but also suggests the mechanisms for its control leading to improved therapeutic interventions.
\end{abstract}

Keywords: Breast cancer; drug resistance; chemotherapy; tamoxifen

\section{Introduction}

Breast cancer (BC) is the most common type of cancer diagnosed in females. It is the second leading cause of mortality in women over the globe. The higher death rate corresponds to the metastatic occurrence of the breast cells invading the primary tissue and then colonizing the distant sites (Ferreira et al., 2020). The stimulation of the female reproductive hormones (including estrogen, progesterone, etc.), specifically during the period of breast development, is perhaps the reason for the increased susceptibility to breast cancer in women as compared to men (Brisken \& O'Malley, 2010).

\section{Epidemiology}

Despite epidemiological and clinical advances in research, there is a continuous rise in the incidence of breast cancer (Qasim et al., 2020). A recent report has shown its impact to be 1 in 20 globally and 1 in 8 in high-income countries (Britt et al., 2020). Female's reproductive history, age, lifestyle patterns, genetic and environmental factors - all have a strong impact on the disease course (Youn \& Han, 2020). Even with high incidence, the early detection and effective chemotherapeutic strategies have helped reducing the rate of mortality and improving the quality of life of the BC patients (Mubarik et al., 2020).

\section{$2.1 \quad$ Sub-types}

The immunohistochemistry (ICH) biomarkers such as estrogen receptor (ER), progesterone receptor (PR) and human epidermal growth factor receptor 2 (HER2 or sometimes called HER2/neu) coupled with the conventional pathological variables including tumor grade, size, lymph node involvement, etc., are strictly considered for diagnosis, prognosis, and disease management in patients (Dai et al., 2015). Based on these receptor interactions and signaling, breast cancer is sub-classified as:

\footnotetext{
* Corresponding author: Asima Tayyeb

School of Biological Sciences, University of the Punjab, Lahore, Pakistan.
}

Copyright $@ 2020$ Author(s) retain the copyright of this article. This article is published under the terms of the Creative Commons Attribution Liscense 4.0. 
- $\quad$ Luminal A: ER (+), HER2/neu (-)

- Luminal B: ER (+), HER2/neu (-)

- HER2/neu rich: ER (-), HER2/neu (+)

- $\quad$ Basal like or triple negative: ER $(-)$, HER2/neu $(-)$

Triple negative breast cancer (TNBC) is the most aggressive form of breast cancer which relates to poor outcomes in patients owing to nodal involvement and markedly increased tumor size (D. Singh et al., 2020). It has been estimated that two-third of the breast cancer (BC) patients are estrogen receptor (ER) positive (García-Becerra et al., 2013). ER is a hormonal receptor, in response to which the mammary epithelial cells are stimulated for uncontrolled proliferation (Jameera Begam et al. 2017).

The binding of estrogen hormone to the target protein- the estrogen receptor (ER) - mediates receptor phosphorylation, brings conformational changes in the active site and triggers receptor dimerization (Shou et al., 2004). This in turn activates transcription by facilitating the binding of the receptor complex to the promoter region of the ER associated genes encoding growth factors and other signaling molecules (Björnström \& Sjöberg, 2005).

Former literature reviews have found that the transcriptional activity of ER is modulated by protein-protein interactions of either co-activators or co-repressors (Shou et al., 2004). In case of breast cancer, the altered receptor conformation promotes transcriptional activation by the active recruitment of co-activators which is a stimulus for cell proliferation (M. Chang, 2012) wrong order should be Chang, 2012. This makes ER receptor a potent target in antiestrogen cancer therapy (Yaşar et al., 2016).

\section{First Line Endocrine Therapy for Breast Cancer}

Endocrine therapy has shown to be effective since decades for breast cancer. Herein, tamoxifen plays its fundamental role in reducing the disease incidence (Awan \& Esfahani. 2018; Gruvberger-Saal et al. 2007). In contrast to the hormonal action, the binding of tamoxifen to the estrogen receptor brings about conformational changes that impedes transcription by the function of co-repressors (Severson et al. 2016).

Fundamental to tamoxifen therapy is the interruption in estrogen signaling either by estrogen receptor blockage or by the competitive reduction in the intracellular amount of estrogen available for receptor binding (Michalides et al., 2004). Therefore, tamoxifen is referred to as selective estrogen receptor modulator (SERM) and is of considerable clinical significance (Johnston. 2010; Zhu et al. 2018).

\section{Metabolism of Tamoxifen}

Tamoxifen is a pro-drug which holds little affinity for its target ER protein (García-Becerra et al. 2013). It requires metabolic activation by CYP gene products which then convert inactive TAM into its active metabolites including 4hydroxy-tamoxifen (4OH-TMX) and N-desmethyl-4-1 hydroxy tamoxifen (endoxifen). These metabolites have more affinity for ER than tamoxifen itself (Ali et al. 2016)

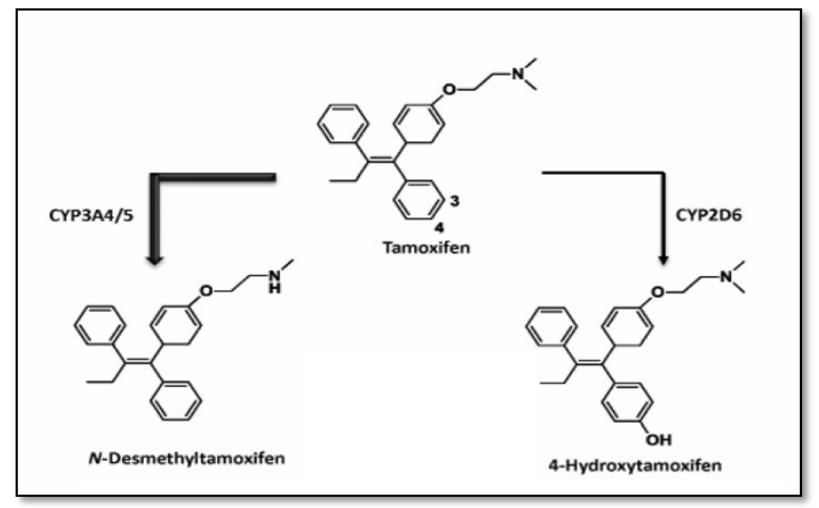


Figure 1 CYP mediated catalysis of tamoxifen into active metabolites in human liver (M. Chang, 2012).

\section{Mechanism of action}

4 hydroxytamoxifen works as an estrogen receptor antagonist by inhibiting the transcription of estrogen responsive genes. This ER/40H-TAM complex recruits NCoR and SMRT - the co-repressor proteins for the regulation of several key genes (Ring \& Dowsett, 2004). Tamoxifen along with PAX2 protein exerts its anti-tumor effects by suppressing the expression of pro-proliferative protein, ERBB2 (Ali et al. 2016).

In addition to anti-cancer activity of TAM, the intrinsic (de novo) or acquired resistance is a major challenge. Despite improved therapeutic strategies, tamoxifen resistance remains a serious clinical challenge (Tremont et al., 2017). The de novo or acquired drug resistance is a significant limiting factor in the anti-estrogen therapy (Desbats et al., 2020). This limited therapeutic efficacy of TAM is determinant of poor prognosis of breast cancer in females and is held accountable for the recurrence or relapse of the disease in 40\% cases (Ali et al. 2016; Zhu et al. 2018).

\section{De novo resistance}

The ER positive breast cancers that establish resistance to the anti-hormonal therapy from the very beginning of the Tam treatment are cases of de novo resistance (Massarweh et al. 2008). Previous studies have demonstrated that MCF$7 \mathrm{ER}+$ breast cancer cell line when transfected with HER2/Neu gene induced cell growth and proliferation in xenograft mice even during the course of tamoxifen therapy. From this in vivo study, it was speculated that the cross talk between growth factor receptor pathways and estrogen receptor contributes well to the development of resistance in the cells (Björnström \& Sjöberg. 2005; Riggins et al. 2007 et al; Shou et al. 2004).

\section{Acquired resistance}

The long-term or continuous therapy with tamoxifen allows the cells that were initially responding to the drug to acquire resistance (Viedma-Rodríguez et al., 2014). Growing evidence indicates that the unresponsiveness to tamoxifen in initially responsive breast tumor leads to breast cancer recurrence in many patients, most probably due to cancer stem cells (CSCs) (Ali et al. 2016). Though majority of the cells are killed but a few cells that evade treatment progress to a resistant phenotype. Many patients who report recurrence respond to a second line therapy (Russell et al. 2007).

A better insight into the molecular basis of resistance mechanisms can provide novel strategies to bypass the limited therapeutic potential of tamoxifen and make advancements in cancer therapeutics (Hultsch et al. 2018).

\section{Mechanisms of Acquired Tamoxifen Resistance}

Due to the complexity of the signal transduction pathways involved, the exact mechanisms underlying acquired resistance remain elusive (Achinger-Kawecka et al. 2020). It is unlikely to say that a single gene or a specific molecular pathway features tamoxifen resistance in patients. Rather there exists a dynamic interplay between various pathways that mark cellular and molecular events leading to drug resistance (Mills et al. 2018).

Furthermore, the mechanisms may vary depending upon breast tumor heterogeneity. Thus, it is a major clinical goal to overcome resistance (Osipo, Gajdos, Cheng, \& Jordan, 2005). various resistance mechanisms have been proposed so far. The most pronounced ones are summarized below (Figure 2) (Chang, 2012) 


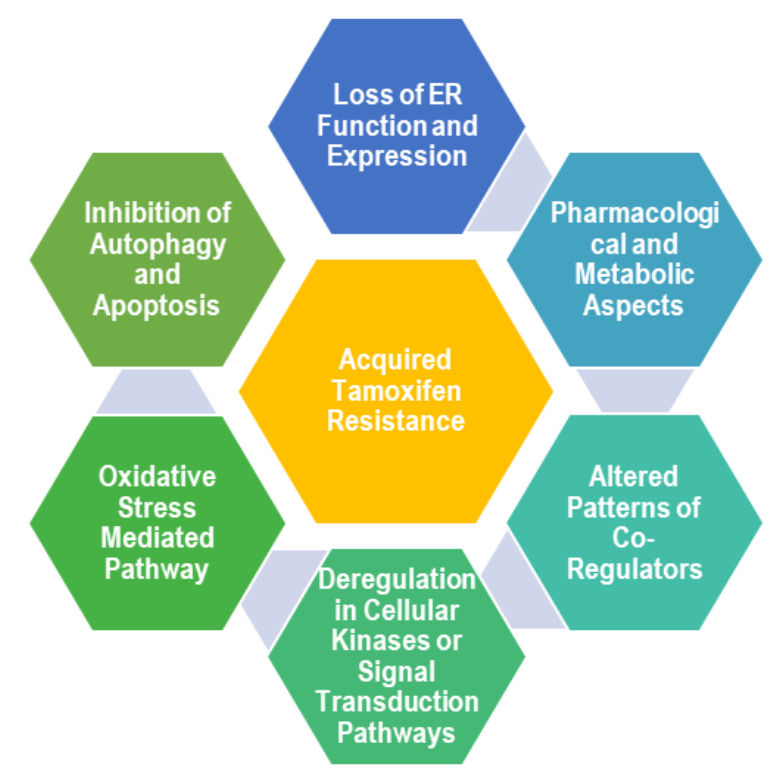

Figure 2 Summary of mechanisms involved in the acquired tamoxifen chemotherapeutic drug resistance of breast cancer.

\subsection{Loss of ER Function and Expression}

The ER antagonistic effect of tamoxifen is primarily based on receptor targeting. Loss of target ER confers resistance to endocrine therapy and contributes to tumorigenesis (Dorssers et al. 2001). Previous studies suggest that loss of ER expression predominates de novo tamoxifen resistance in cells (Clarke et al., 2003).Interestingly, loss of ER results in the change in phenotype from ER positive to ER negative (Chang, 2012). This may be attributed to the transcriptional inhibition of ER genes (Lewis \& Jordan, 2005).

Epigenetic modification such as histone deacetylation by HDAC (histone deacetylases) or hypermethylation of CpG islands by DNA methyltransferase (DNMT) is another major event that causes the silencing of ER genes, thereby reducing ER expression (H. G. Chang et al., 2005) order. It has been well documented that ER specific miRNAs play an endogenous effector role in RNA interference and repress the mRNA translation of ER-alpha subdomain (Chang, 2012) order. Hyper-activation of mitogen-activated protein kinase (MAPK) is another regulatory mechanism for induction of ER negativity in endocrine resistant cells.

Some other factors that resulting in the loss of ER expression include mutations and abnormal splicing. It remains unclear whether ER mutations are of any clinical significance or not (Wang et al., 2019). However, resistance to antiestrogen therapy can even be developed in the absence of any apparent gene mutation. The splicing variants are yet to be determined for their relevance to chemotherapeutic resistance (Viedma-Rodríguez et al., 2014).

\subsection{Pharmacological and Metabolic Aspects}

Marked reduction in the intracellular drug concentration is another potential resistance mechanism. This is partly due to the increased efflux or decreased influx; the former being linked to the overexpression of P glycoprotein (Pgp). Pgp is a $170 \mathrm{kDa}$ ATP-driven membrane pump that expels the cytotoxic drug using the energy released by ATP hydrolysis. It is still vague that to what extent this resistance mechanism works to saturate ER and lower TAM efficacy. The increase in the metabolic potential of tamoxifen to estrogenic metabolites further contributes to resistance development in patients diagnosed with breast cancer. Emerging evidence shows that N-desmethyltamoxifen is the major antiestrogenic metabolite present in the serum. Conversely, the levels of 4-hydroxytamoxifen (4-OH TAM) in serum are low yet the binding affinity of 4-OH TAM is much greater than that of tamoxifen. The generation of this hydroxylated product is CYP2D6 dependent.

Patients having a wild type allele of CYP2D6 were co-administered with paroxetine and tamoxifen, and they exhibited decreased plasma levels of 4-OH TAM. Alongside, women who underwent TAM therapy and carried a variant allele of CYP2D6 also had lower concentrations of this TAM metabolite. The results of this study illustrated that the pharmacological interactions as well as drug pharmacogenomics serve to decrease TAM efficiency in breast cancer cells. Single nucleotide polymorphism (SNP) in CYP2D6 is another factor responsible for the null or minimal tamoxifen 
response to cancer cells (Viedma-Rodríguez et al., 2014). This initiates competition between estrogenic and antiestrogenic metabolites for ER activation. As per previous studies, a huge amount of estrogen will be needed to diminish the anti-estrogenic effects of tamoxifen. Furthermore, it has been observed that the concentration of TAM metabolites in the serum remains constant for several years following therapy.

\subsection{Altered Patterns of Co-Regulators}

Co-activators and co-repressors have dominating roles in context of ER. It has been predicted that aberrant expression of co-regulators can potentially lead to a tamoxifen resistant phenotype.

\subsubsection{Co-activators}

AIB1 (amplified in breast cancer 1) is a member of steroid receptor co-activator family (SRC). It interacts in a ligand dependent fashion and its overexpression constitutes $50 \%$ of the breast tumors. In vitro and in vivo studies have also indicated its up-regulation in MCF-7 cell line (Haque \& Desai, 2019). Thus, high AIB1 in humans and cultured cells are indicative of enhanced agonist activity of chemotherapeutic agent TAM (Osborne \& Schiff, 2003).

\subsubsection{Co-repressors}

Upon recruitment of co-repressor proteins on ER, multi- subunit repressor complexes are formed which involve HDACs for the condensation of chromatin and for transcription repression. Notably, co-repressors such as NCoR1and NCoR2 are conditionally recruited, provided that an antagonist (tamoxifen) has pre-formed a complex with ER. Consequently, the agonist activity will be inhibited. This implies that the minimal effects of TAM therapy may in turn be due to the progressive decrease in the co-repressor activity. Low protein levels of NCoR point towards poor prognostic value due to which the cells are subjected to acquire resistance in both in vitro and in vivo models (Osborne \& Schiff, 2003).

\subsection{Deregulation in Cellular Kinases or Signal Transduction Pathways}

The mechanistic behavior of estrogen receptor cannot be studied in isolation from other signal cascade pathways. It is critical to study the dynamic regulatory interactions concerning ER, growth factors and signal transduction pathways for a better understanding (Osborne \& Schiff, 2003).

\subsubsection{Growth factor signaling}

It has been evidenced that there occurs a cross talk between estrogen receptor and growth factor receptor pathways; most importantly those of epidermal growth factor receptor - EGFR/HER2 and insulin-like growth factor receptor (IGFR) families (Gururaj et al., 2006).ER has two activation function (AF) domains, AF-1 and AF-2 (Puranik et al., 2019). ERK1 and ERK2 of the MAPK family modulate the expression regulation by phosphorylating ER at serine 118 position within AF-1 domain. This increases the likelihood of ER for ligand and activates ER directly - independent of ligand (Okat, 2018).Besides, serine 167 is also phosphorylated in AF-1 domain of ER through the action of ribosomal S6 kinase (RS6) which is itself activated by ERK1 and ERK2. This means that increase in the expression of ERK1/2 can possibly confer resistance to anti-estrogen regimen (Shou et al., 2004).

Additionally, growth factor signaling does have an indirect impact on ER activation by stimulating the co-activator response and impairing the co-repressor response (Nicholson et al., 2007). Such responses are believed to be achieved by phosphorylating transcriptional co-regulators which influences their nuclear sub-localization (Awan \& Esfahani, 2018). It is noteworthy that up-regulation in peptide growth factor signaling pathway during endocrine therapy is a clear indication of Tam resistance (Moerkens et al., 2014).

Recent studies investigated the role of IGFR and EGFR in conferring resistance to the cells (Chong et al., 2011) et al. Ligand- receptor complex physically and directly relates to the activation of IGFR and is associated with downstream ERK1/2 MAPK signaling cascade (Ali et al., 2016).Membrane bound ER also has a direct physical association with HER2 and the transactivation of EGFR by phosphorylation (Davoli et al., 2010). Higher expression of EGFR/HER2 in MCF7 cell line enables cell proliferation and inhibits cellular apoptosis. This is another approach by which cells acquire resistance to tamoxifen (Fan et al., 2015).

\subsection{Role of Oxidative Stress Mediated Pathway}

The interaction of ER with stress-activated protein kinase/c-junNH2 terminal kinase pathway is important to comprehend resistance in a stress-induced environment. AP-1 is a transcriptional complex of Fos and Jun whose binding to DNA at AP-1 response element is initiated by dimerization. AP-1 transcription is enhanced in response to phosphorylation of these components by junNH2 terminal kinases (JNKs) or stress activated protein kinases (SAPKs). 
Administration of tamoxifen for a longer time may create intracellular oxidative stress. Under such conditions, these enzymes are activated. Cells that manifest a rise in AP-1 activity are thought to have developed TAM resistance. Elevation in the oxidative stress may result in a more aggressive resistant phenotype.

\subsection{P38 MAPK regulation}

P38 MAPK activation has also been reported in case of extracellular stimuli detection such as chemical or physical stress, cytokines, etc. This pathway is switched on in the cell lines that express ER and 4-OH TAM eventually causes apoptosis.

Tamoxifen-induced stress negatively affects the signaling of p38 MAPK. Due to the inhibition of this pathway, 4-OH TAM fails to induce apoptosis and the cells tend to resist anti-cancer therapy. Peroxiredoxins (prxns) are the anti-oxidant modulators for intracellular redox cycling. They are known for protecting cells from oxidative damage by the modulation of apoptosis. Prx5 is found in various locations where reactive oxygen species (ROSs) may generate such as in mitochondria, cytosol, peroxisomes, etc. The expression of prx 5 is due to ROS. Higher expression of prx 5 has been reported in breast cancer tissues. Thus, this anti-oxidant has role in mammary tumorigenesis.

GATA-1 has shown to repress the transcription of prx 5 and this correlates with agonist bound ER activity. This makes Tam inefficient as it loses its ability to prevent the repression of anti-oxidant, prx 5 protein. Cells will continue to grow despite TAM treatment because of apoptotic suppression.

\subsection{Inhibition of Autophagy and Apoptosis}

Autophagy refers to self-eating. It is a mechanism for the digestion of cytoplasmic contents including misfolded or unfolded proteins, sub-cellular organelles by the formation of autophagosomes. It is a way to restore homeostasis under stress conditions. Clinical research has figured out the role of autophagy and apoptosis in resistant breast cancer. Overexpression of beclin-1, an important biomarker for autophagy, tends to reduce tamoxifen sensitivity to cancer cells.

\subsection{Role of Cancer Stem Cells (CSCs)}

The cells having stem-cell like properties constitute a sub-population that can sustain tumorigenesis are termed as cancer stem cells (CSCs) (Raman, Tiwari, Tiriveedhi, \& Rhoades 2020) et al. They share the properties of self-renewal and differentiation with normal stem cells present in the body. CSCs when seeded into an animal host can trigger carcinogenesis (Butti et al., 2019).

Presumably, CSCs may emerge from the reactivation of the senescent cells that are in cell-cycle arrest. Senescent cells suffer from energy deprivation and can be revived by supplementing the cell culture media with NADH precursors (Sotgia et al., 2019). CSCs strictly rely on NADH for their propagation and the over-expression of numerous embryonic markers of stemness such as oct4, c-myc has been investigated in pre-clinical and clinical settings (Gwak et al., 2017).

The stem cells in a normal breast possess the basal phenotype and they are dependent on EGFR and some other growth factor receptor molecules (O'Brien et al., 2009). Breast cancer stem cells (BCSCs) are involved in acquisition of resistance to Tam. In resistant breast tumors, the population of BCSCs expands (Zhou et al., 2019). Enrichment of BCSCs during anti-estrogenic treatment has a central contribution in drug resistance and this has been confirmed in MCF-7 Tam resistant cell line (Liu et al., 2013).

\section{Conclusion}

Accumulating data on different mechanisms involved in tamoxifen resistance suggest that they can be targeted to eradicate drug resistance and improve therapeutic interventions. However, the present knowledge is limited to research mostly on in vitro models, therefore, further research with large animal models and clinical samples can provide concrete basis for the control of chemotherapeutic drug resistance.

\section{Compliance with ethical standards}

\section{Acknowledgments}

The author acknowledges all the scientists working and tried to elucidate the different mechanisms involved in the complex field of chemotherapeutic drug resistance. 


\section{Disclosure of conflict of interest}

The author has no conflict of interest regarding the preparation and publication of this manuscript.

\section{References}

[1] Achinger-Kawecka J, Valdes-Mora, F, Luu PL, Giles KA, Caldon CE, Qu W, Clark SJ. Epigenetic reprogramming at estrogen-receptor binding sites alters 3D chromatin landscape in endocrine-resistant breast cancer. Nature Communications. 2020; 11(1): 320.

[2] Ali S, Rasool M, Chaoudhry HN, Pushparaj P, Jha P, Hafiz A. Sarwar Jamal, M. Molecular mechanisms and mode of tamoxifen resistance in breast cancer. Bioinformation. 2016; 12(3): 135-139.

[3] Awan, A, Esfahani K. Endocrine therapy for breast cancer in the primary care setting. Current oncology (Toronto, Ont.). 2018; 25(4): 285-291.

[4] Björnström L, Sjöberg, M. Mechanisms of Estrogen Receptor Signaling: Convergence of Genomic and Nongenomic Actions on Target Genes. Molecular Endocrinology 2005; 19(4): 833-842.

[5] Brisken C, O'Malley B. Hormone action in the mammary gland. Cold Spring Harbor perspectives in biology. 2010; 2(12): a003178-a003178.

[6] Britt KL, Cuzick J, Phillips KA. Key steps for effective breast cancer prevention. Nature Reviews Cancer. 2020; 20(8): 417-436.

[7] Butti R, Gunasekaran VP, Kumar TVS, Banerjee P, Kundu GC. Breast cancer stem cells: Biology and therapeutic implications. The International Journal of Biochemistry \& Cell Biology. 2019; 107: 38-52.

[8] Chang HG, Kim SJ, Chung KW, Noh DY, Kwon Y, Lee ES, Kang HS. Tamoxifen-resistant breast cancers show less frequent methylation of the estrogen receptor $\beta$ but not the estrogen receptor $\alpha$ gene. Journal of Molecular Medicine. 2005; 83(2): 132-139.

[9] Chang, M. Tamoxifen resistance in breast cancer. Biomolecules \& therapeutics. 2012; 20(3): $256-267$.

[10] Chong K, Subramanian A, Sharma A, Mokbel K. Measuring IGF-1, ER- $\alpha$ and EGFR expression can predict tamoxifen-resistance in ER-positive breast cancer. Anticancer Res. 2011; 31(1): 23-32.

[11] Clarke R, Liu MC, Bouker KB, Gu Z, Lee RY, Zhu Y. Hilakivi-Clarke, L. A. (2003). Antiestrogen resistance in breast cancer and the role of estrogen receptor signaling. Oncogene. 22(47): 7316-7339.

[12] Dai X, Li T, Bai Z, Yang Y, Liu X, Zhan J, Shi B. Breast cancer intrinsic subtype classification, clinical use and future trends. American journal of cancer research. 2015; 5(10): 2929-2943.

[13] Davoli A, Hocevar BA, Brown TL. Progression and treatment of HER2-positive breast cancer. Cancer Chemotherapy and Pharmacology. 2010; 65(4): 611-623.

[14] Desbats MA, Giacomini I, Prayer-Galetti T, Montopoli M. Metabolic Plasticity in Chemotherapy Resistance. Frontiers in Oncology. 2020;10(281).

[15] Dorssers LCJ, van der Flier S, Brinkman A, van Agthoven T, Veldscholte J, Berns EMJJ. Foekens JA. (2001). Tamoxifen Resistance in Breast Cancer. Drugs. 2001; 61(12): 1721-1733.

[16] Fan W, Chang J, Fu P. Endocrine therapy resistance in breast cancer: current status, possible mechanisms and overcoming strategies. Future medicinal chemistry. 2015; 7(12): 1511-1519.

[17] Ferreira LC, Orso F, Dettori D, Lacerda JZ, Borin TF, Taverna D, Zuccari DAPC. The role of melatonin on miRNAs modulation in triple-negative breast cancer cells. PloS one. 2020; 15(2): e0228062.

[18] García-Becerra R, Santos N, Díaz L, Camacho J. Mechanisms of resistance to endocrine therapy in breast cancer: focus on signaling pathways, miRNAs and genetically based resistance. International journal of molecular sciences. 2013; 14(1): 108-145.

[19] Gruvberger-Saal SK, Bendahl PO, Saal LH, Laakso M, Hegardt C, Edén P, Fernö M. Estrogen Receptor $\beta$ Expression Is Associated with Tamoxifen Response in ER $\alpha$-Negative Breast Carcinoma. Clinical Cancer Research. 2007 ; 13(7): 1987-1994.

[20] Gururaj AE, Rayala SK, Vadlamudi RK, Kumar R. Novel Mechanisms of Resistance to Endocrine Therapy: Genomic and Nongenomic Considerations. Clinical Cancer Research. 2006; 12(3): 1001s-1007 
[21] Gwak JM, Kim M, Kim HJ, Jang MH, Park SY. Expression of embryonal stem cell transcription factors in breast cancer: Oct4 as an indicator for poor clinical outcome and tamoxifen resistance. Oncotarget. 2017; 8(22): 3630536318.

[22] Haque MM, Desai KV. Pathways to Endocrine Therapy Resistance in Breast Cancer. Frontiers in Endocrinology. 2019; 10(573).

[23] Hultsch S, Kankainen M, Paavolainen L, Kovanen RM, Ikonen E, Kangaspeska S. Kallioniemi O. Association of tamoxifen resistance and lipid reprogramming in breast cancer. BMC Cancer. 2018; 18(1): 850.

[24] Jameera Begam A, Jubie S, Nanjan MJ. Estrogen receptor agonists/antagonists in breast cancer therapy: A critical review. Bioorganic Chemistry. 2017; 71: 257-274.

[25] Johnston SRD. New Strategies in Estrogen Receptor-Positive Breast Cancer. Clinical Cancer Research. 2010; 16(7): 1979-1987.

[26] Lewis JS, Jordan VC. Selective estrogen receptor modulators (SERMs): Mechanisms of anticarcinogenesis and drug resistance. Mutation Research/Fundamental and Molecular Mechanisms of Mutagenesis. 2005; 591(1): 247-263.

[27] Liu H, Zhang HW, Sun XF, Guo XH, He YN, Cui SD, Fan QX. Tamoxifen-resistant breast cancer cells possess cancer stem-like cell properties. Chin Med J (Engl). 2013: 126(16): 3030-3034.

[28] Massarweh S, Osborne CK, Creighton CJ, Qin L, Tsimelzon A, Huang S, Schiff R. (2008). Tamoxifen Resistance in Breast Tumors Is Driven by Growth Factor Receptor Signaling with Repression of Classic Estrogen Receptor Genomic Function. Cancer Research. 2008; 68(3): 826-833.

[29] Michalides R, Griekspoor A, Balkenende A, Verwoerd D, Janssen L, Jalink K, Neefjes J. Tamoxifen resistance by a conformational arrest of the estrogen receptor $\alpha$ after PKA activation in breast cancer. Cancer Cell. 2004; 5(6): 597-605.

[30] Mills JN, Rutkovsky AC, Giordano A. Mechanisms of resistance in estrogen receptor positive breast cancer: overcoming resistance to tamoxifen/aromatase inhibitors. Current Opinion in Pharmacology. 2018; 41: 59-65.

[31] Moerkens M, Zhang Y, Wester L, van de Water B, Meerman JHN. Epidermal growth factor receptor signalling in human breast cancer cells operates parallel to estrogen receptor $\alpha$ signalling and results in tamoxifen insensitive proliferation. BMC Cancer. 2014; 14: 283-283.

[32] Mubarik S, Wang F, Fawad M, Wang Y, Ahmad I, Yu C. Trends and Projections in Breast Cancer Mortality among four Asian countries (1990-2017): Evidence from five Stochastic Mortality Models. Scientific Reports. 2014; 10(1): 5480.

[33] Nicholson R, Hutcheson I, Jones H, Hiscox S, Giles M, Taylor K, Gee J. Growth factor signalling in endocrine and anti-growth factor resistant breast cancer. Reviews in endocrine \& metabolic disorders. 2007; 8: 241-253.

[34] O’Brien CS, Howell SJ, Farnie G, Clarke RB. Resistance to Endocrine Therapy: Are Breast Cancer Stem Cells the Culprits? Journal of Mammary Gland Biology and Neoplasia. 2009; 14(1): 45-54.

[35] Okat Z. Molecular dynamics of estrogen receptors. Eurasian Journal in Medicine and Oncology. 2018; 2(4); 189197.

[36] Osborne CK, Schiff R. Growth factor receptor cross-talk with estrogen receptor as a mechanism for tamoxifen resistance in breast cancer. The Breast. 2003; 12(6): 362-367.

[37] Osipo C, Gajdos C, Cheng D, Jordan VC. Reversal of tamoxifen resistant breast cancer by low dose estrogen therapy. The Journal of Steroid Biochemistry and Molecular Biology. 2005; 93(2): 249-256.

[38] Puranik NV, Srivastava P, Bhatt G, John Mary DJS, Limaye AM, Sivaraman J. Determination and analysis of agonist and antagonist potential of naturally occurring flavonoids for estrogen receptor (ER $\alpha$ ) by various parameters and molecular modelling approach. Scientific Reports. 2019; 9(1): 7450.

[39] Qasim S, Tayyab H, Zulqadar K, Masood S, Qasim TB, Zubair Z. Breast Cancer knowledge and perceived barriers to help seeking among pre-clinical and clinical female medical students of King Edward Medical University, Lahore: a cross-sectional study. BMC Medical Education. 2020; 20(1): 222.

[40] Raman D, Tiwari AK, Tiriveedhi V, Rhoades JA. Editorial: The Role of Breast Cancer Stem Cells in Clinical Outcomes. Frontiers in Oncology. 2020; 10(299). 
[41] Riggins RB, Schrecengost RS, Guerrero MS, Bouton AH. Pathways to tamoxifen resistance. Cancer letters. 2007; 256(1): 1-24.

[42] Ring A, Dowsett M. Mechanisms of tamoxifen resistance. Endocr Relat Cancer. 2004; 11(4): 643-658.

[43] Russell D, Clark K, deGraffenried L, James F, Beeram M. <em $>$ In vitro</em> modeling of acquired tamoxifen resistance. Cancer Research. 2007; 67(9 Supplement): 999-999.

[44] Severson TM, Nevedomskaya E, Peeters J, Kuilman T, Krijgsman 0, van Rossum A, Zwart W. Neoadjuvant tamoxifen synchronizes ER $\alpha$ binding and gene expression profiles related to outcome and proliferation. Oncotarget. 2016; 7(23): 33901-33918.

[45] Shou J, Massarweh S, Osborne CK, Wakeling AE, Ali S, Weiss H, Schiff R. Mechanisms of Tamoxifen Resistance: Increased Estrogen Receptor-HER2/neu Cross-Talk in ER/HER2-Positive Breast Cancer. JNCI: Journal of the National Cancer Institute. 2004; 96(12): 926-935.

[46] Sotgia F, Fiorillo M, Lisanti MP. Hallmarks of the cancer cell of origin: Comparisons with "energetic" cancer stem cells (e-CSCs). Aging. 2019; 11(3) 1065-1068.

[47] Tremont A, Lu J, Cole JT. Endocrine Therapy for Early Breast Cancer: Updated Review. The Ochsner journal. 2017 17(4): 405-411.

[48] Viedma-Rodríguez R, Baiza-Gutman L, Salamanca-Gómez, F, Diaz-Zaragoza M, Martínez-Hernández G, Ruiz Esparza-Garrido R, Arenas-Aranda D. Mechanisms associated with resistance to tamoxifen in estrogen receptorpositive breast cancer (Review). Oncol Rep. 2014; 32(1): 3-15.

[49] Wang Q, Gun M, Hong Xy. Induced Tamoxifen Resistance is Mediated by Increased Methylation of E-Cadherin in Estrogen Receptor-Expressing Breast Cancer Cells. Scientific Reports. 2019; 9(1): 14140.

[50] Yaşar P, Ayaz G, User SD, Güpür G, Muyan M. Molecular mechanism of estrogen-estrogen receptor signaling. Reproductive medicine and biology. 2016; 16(1): 4-20.

[51] Youn HJ, Han W. (2020). A Review of the Epidemiology of Breast Cancer in Asia: Focus on Risk Factors. Asian Pacific Journal of Cancer Prevention. 2020; 21(4): 867-880.

[52] Zhou J, Chen Q, Zou Y, Chen H, Qi L, Chen Y. Stem Cells and Cellular Origins of Breast Cancer: Updates in the Rationale, Controversies, and Therapeutic Implications. Frontiers in Oncology 2019; 9: 820-820.

[53] Zhu Y, Liu Y, Zhang C, Chu J, Wu Y, Li Y, Liu Q. Tamoxifen-resistant breast cancer cells are resistant to DNAdamaging chemotherapy because of upregulated BARD1 and BRCA1. Nature Communications. 2018; 9(1): 1595. 